\title{
Remote maintenance system for inspection robot based on CDMA network
}

\author{
Dezheng Zhao, En Li, Guodong Yang, Zize Liang \\ State Key Laboratory of Management and Control for Complex Systems, \\ Institute of Automation, Chinese Academy of Sciences \\ Beijing, China \\ \{Dezheng.zhao, En.li, Guodong.yang, Zize.liang\}@ia.ac.cn
}

\begin{abstract}
In order to remotely maintain the robots for high voltage power transmission lines in field application, a remote robot maintenance system is built up based on CDMA network. Network connection between the robot and remote maintenance center is established through intranet within CDMA network. During the communication process, data transferred are encoded / decoded with a specially designed protocol to improve the stability and security of data transmission. After application experiments reveal that remote maintenance system can obtain real-time operation information of robot and line inspection results and line inspection results, conduct remote location of GPS device on robot, and get the robot position and line fault position to provide technical basis for network management of operation of inspection robot.
\end{abstract}

Keywords-Power transmission line; Inspection robot; CDMA network; Remote maintenance

\section{INTRODUCTION}

High voltage and extra high voltage overhead power line is the main way of long-distance power transmission. Its operational safety must be reliably guaranteed. The power companies need to inspect the transmission lines to obtain the operation status on regular basis, and detect and eliminate security risks, in order to guarantee the normal operation of transmission lines.

At present, there are two main transmission line inspection methods, including ground visual method and aerial method. But they have their own inherent defects in terms of work efficiency, technical difficulty and operating costs $[1,2]$. In recent years, high voltage power transmission line inspection robot [3-5] gradually becomes one of the research hotspots in high voltage line inspection field. This robot can walk quickly on the transmission line with its self-navigation, and can overcome such various obstacles as strain clamp, anti-vibration hammer, suspension clamp and so on. Meanwhile, the robot carries all kinds of sensor, GPS positioning device, and transmission line detection equipment, used for real-time online detection of operation status of transmission line.

When the robot is running in the complex field environment, the operator can't effectively control it closely. It must be built a set of robot remote maintenance system, through which operator can obtain the operation status of all robots working at the inspection scene and control them in real-time [6, 7].

This paper introduces an inspection robot remote maintenance system based on CDMA network. Firstly, the system architecture is described. Secondly, in view of the characteristics of the CDMA network, networking scheme by using of establishing connection in the intranet is proposed. And then considering the stability and safety of data transmission, a unified protocol is designed for data encoding and decoding. Finally, the running experiment on this system is conducted and the experimental results are analyzed.

\section{SYSTEM ARCHITECTURE}

The system is mainly composed of maintenance center server, multiple inspection robot, and wireless module for wireless data transmission communication that is connected with the maintenance center server and inspection robot. Thus constitute a distributed monitoring and detection network based on CDMA wireless public network as shown in Figure 1. Maintenance center server and inspection robot are connected with CDMA network through wireless module. In this way a network connection for data transmission can be established between the maintenance center server and inspection robot within the same network. In order to ensure the stability of the telecommunication data, TCP connection is adopted to transfer data. Maintenance center server can manage multiple robots and distinguish different robots by phone number as the only identification bound with robot wireless module.

Inspection robot control system is designed in accordance with the hierarchical structure. It includes management level, the organization level, the coordination level and the execution level. The structure is shown in Figure 2. Management level is the uppermost layer of the entire system. It is the interface the operator controlled and maintained the robots. Organizational level is the core of the entire system of control. It operates the entire system to organize, manage, and interact with the outside world. Coordination level is composed of various subsystems control module. It is the interface interacted between the central controller and peripheral instrument. Executive level is the lowest level of the entire system, which is responsible for the concrete implementation of the various tasks.

Remote maintenance software is not only the core of maintenance terminals but also the interface of human-computer interaction. Its system framework is shown in Figure 3. Remote maintenance software can implement such functions as robot basic information input, show and query the robot historical data, the remote read and store 
historical data, display real-time data, GPS positioning of the robot. The software system adopts a typical three-tier architecture [8], including the presentation layer, business logic layer and data access layer. The individual layers are mutually independent. The presentation layer is designed to mainly complete the task of human-computer interaction. Business logic layer is the actual implementation of the system operation. The data access layer is the bottom of the system. It is used to store data information. With layered system structure, it can decouple the implementation of the system in logic, and thus facilitate code maintenance and function extension.

\section{COMMUNICATION MODE}

With respect to the Internet, CDMA network is a semi-closed private network. The IP address obtained when user dial-up Internet is a private address dynamically allocated by the telecom operators and indeed not be open to the public. Therefore, CDMA network is a LAN within the Internet. In order to achieve external active access to the LAN, port mapping mechanism must be established. However, port mapping mechanism between CDMA and the Internet is also decided by the telecom operators. It is not open to the public too. Thus the network connection is only established from the inside to the outside of the CDMA network, not the other way round. So it can't be achieved that maintenance center direct access to the robots. With the dwindling of IPv4 addresses, many companies and organizations are using NAT (Network Address Translation) technology [9] to connect the public network, resulting in the maintenance center servers are often put in a private network. Similarly, network devices in the public network can't direct access the network devices in a private network, let alone network equipment in the private CDMA network direct access to other private network equipment. Therefore, networking solutions for the use of the robot to take the initiative to access the maintenance center server can't be achieved.

The rebound port Trojan [10] communication technology can connect the robots and maintenance center. That is, the server with a fixed public IP address is used as a hub. The robots within CDMA network and maintenance center in the Internet subnet are connected with the hub. The hub forwards communication packet between the robots and maintenance center. However, this solution needs the robots to be always connected by the hub, regardless of whether the robots need the remote maintenance inspection by maintenance center. The robots remain connection with the hub which results in the less flexibility. And the wireless module is always active. In this way, robot will speed up the power loss. Meanwhile, in the environment of IPv4 address resources nearly depleted, the cost of use a fixed IP address of the public network is also very high.

Taking the above points into account, the system uses CDMA intranet networking to connect the maintenance center and the robots. By the wireless modules dialing, a dynamically assigned IP address is obtained and then the address is sent to the other party in the form of the text messages. Due to the maintenance center and the robots are in the same internal network, thus to establish a connection with each other will not be a problem. Networking program is shown in Figure 4. Networking needs the following steps:

1) To dial by the maintenance center server to establish PPP (Point to Point Protocol) connection with the base station, at the same time to get a dynamically assigned IP address, and then to create the Socket to wait for the robots sending a connection request.

2) The maintenance center server sends the IP address to the robots in the form of the text messages after the successful dial-up access to the Internet.

3) After receiving the text messages sent by the maintenance center server, the robots parse out the IP address and to establish PPP connection with the base station.

4) Robot initiates a connection request. As a Client the robots create the Socket to send connection request for the maintenance center server after the robots successfully on line.

5) The maintenance center server establishes network connection with the robots. After receiving connection request initiated by the robots, maintenance center server will establish a network connection with the robots.

\section{DATA TRANSMISSION}

Since the data is transmitted based on the TCP connection, TCP is connection-oriented and stream-oriented and can provide reliable data transmission services in both transmitter and receiver (client and server). In order to effectively send multiple data packets to the other side, the transmitting terminal uses the optimization method (Nagle algorithm) and combines multiple small data package with short interval into a large data block, packet them, and then send out. Yet since there is no boundary between the data packages in the data block, the receiving terminal will not be able to distinguish the original data packages, resulting in TCP data adhesion.

To overcome the problem of TCP data adhesion and ensure the stability and security of the data transmission, the data transferred need to be encoded and decoded, and the terminals of sending and receiving need to be encoded and decoded with the unified protocol. Therefore, the transmission data package is encapsulated into the data frames in data transmission of the system. A complete data frame contains the frame header, frame end, and frame data as shown in Figure 5. Among them, frame header and frame end together make up the data frame boundary and frame data used to store the data or command.

In the process of data communication, it will inevitably produce errors. It needs to verify the data frames received in order to avoid obtaining wrong information resulting from parse erroneous data. Therefore, CRC 16 bit checksum is added at the end of the data frame. After data frame is received, it will be checked the CRC. If the validation is successful, the data frame will be directly decoded. Otherwise, the data frame will be retransmitted by sending request to the other transmitting terminal. By adopting CRC mode, it greatly improves the stability of the data transmission, and also avoids wrong operation resulting from communication error. 


\section{EXPERIMENT AND ANALYSIS}

The remote maintenance system is constructed based on the program and the experiment is carried out to test it. After the remote maintenance software is connected with the robots, wireless data transmission communication delay is tested with the assistance of remote maintenance software. The remote maintenance software makes timing from issuing data requests to receiving the correct results and gives delay output. By sending the requests from the different size of the data, statistics on the amount of communication data and communication delays is made to obtain the relationship between the two as shown in Figure 6, wherein the abscissa is the size of the transmission data, in bytes while the ordinate is a communication delay time, in seconds.

From the statistics, with the increase in the amount of data transmitted, the communication delay increases, most of the robot remote maintenance of communication delays are maintained in less than 2s. There are too many communication parts appear in the communication process, and it would inevitably produce delay between parts. These delays add up, eventually leading communication delay of the entire system to reaching the second level. On the other hand, unstable signal of the wireless communication itself also makes the communication delay further increase, which is apparent from the chart. There are several mutations of delay because of the wireless signal suddenly becoming weak. Due to the system does not always require very high real-time capability, so the communication delay is entirely acceptable for remote maintenance of the robots. Besides, in the process of remote maintenance of the robots, most operations are to send the command to the robots and get the status information of the robots. Data to be transferred in one operation is relatively small. As for transmission of smaller data, the communication system can ensure a certain real-time capability.

Statistics on the number of wireless communication packet loss is made, and the result is shown in Figure 7, wherein the abscissa is the number of transport packets, and the ordinate for the packet loss number. Too many parts of wireless communication system and unstable signal result in too much packet loss, as is shown in box curve. The packet loss rate is $5.46 \%$. The packet loss rate is too high for remote maintenance of the robots, thus can't meet the communication requirements. To this end, retransmission mechanism is introduced in communication system, the communication sides adopt response mode to transmit data. When one side can't receive response data within a certain time, it will immediately request the other side to retransmit data. Statistics on packet loss with retransmission mechanism is shown in the circle curve. The packet loss rate is only $0.24 \%$. From the statistics, the packet loss rate with retransmission mechanism is greatly reduced, which can ensure the stability of the system of wireless data communication.

From the data analysis of the experimental results, the data communication can meet the real-time requirements of the robot remote maintenance in the remote maintenance process; in the data transmission process, it can ensure the accuracy and stability of the data transmission from adopting mechanism of retransmission when packet loss. The system operation results show that the remote maintenance system can remotely get the robots' running state, read the historical data of robot sensors, locate the robot accurately, remotely control the robot, and thus be able to complete basic remote maintenance tasks for inspection robot.

\section{CONCLUSION}

According to the practical application of power transmission line inspection robot, a complete design scheme of remote maintenance is proposed, used for remote maintenance and management of the robot working in the field environment. This system adopts way of the text message and wireless Internet access to establish a communication channel for wireless data transmission communication. It avoids the problem of conventional data transmission module depending on the fixed public IP address, and doesn't need public network server with fixed IP address for data transfer. Thus it can improve the flexibility of the communication system, and ensure the reliability of data communication.

Using the system, the operator can remotely maintain the inspection robots working in the field environment at anytime and anywhere, and obtain the inspection results of transmission lines from the robots in real-time. Through the GPS positioning function of the system, the operator can get the position of the robots, and locate the transmission line fault point the robots have inspected. In order to send line maintenance staffs to accurately reach the fault location for repair.

After several actual operating of the remote maintenance system, it is proved that the system can complete well the basic remote maintenance work for inspection robot.

\section{ACKNOWLEDGMENT}

This work is supported by the National High Technology Research and Development Program of China(863 Program)( 2012AA041402-1)

\section{REFERENCES}

[1] G. Jaensch, H. Hoffmann, and A. Markees, "Locating defects in high voltage transmission lines," in Transmission \&amp; Distribution Construction, Operation \&amp; Live-Line Maintenance Proceedings, 1998. ESMO '98. 1998 IEEE 8th International Conference on, 1998, pp. 179-186.

[2] C. C. Whitworth, A. W. G. Duller, D. I. Jones, and G. K. Earp, "Aerial video inspection of overhead power lines," Power Engineering Journal, vol. 15, pp. 25-32, 2001.

[3] J. Sawada, K. Kusumoto, Y. Maikawa, T. Munakata, and Y. Ishikawa, "A mobile robot for inspection of power transmission lines," Power Delivery, IEEE Transactions on, vol. 6, pp. 309-315, 1991.

[4] S. Peungsungwal, B. Pungsiri, K. Chamnongthai, and M. Okuda, "Autonomous robot for a power transmission line inspection," in Circuits and Systems, 2001. ISCAS 2001. The 2001 IEEE International Symposium on, 2001, pp. 121-124 vol. 2.

[5] L. Tang, L. Fang, and H. Wang, "Development of an inspection robot control system for $500 \mathrm{KV}$ extra-high voltage power transmission lines," in SICE 2004 Annual Conference, 2004, pp. 1819-1824 vol. 2.

[6] L. Zi-ze, L. En, T. Min, G. P. Liu, and D. Rees, "Control of Inspection Robots for Power Transmission Lines Based on Remote Video 
over Internet," in Computational Intelligence for Modelling, Control and Automation, 2005 and International Conference on Intelligent Agents, Web Technologies and Internet Commerce, International Conference on, 2005, pp. 877-882.

[7] C. Truche, M. Mokhtari, and C. Vallet, "Telediagnosis and remote maintenance system through the Internet for the Manus robot," Assistive Technology on the Threshold of the New Millennium, Bühler, C. and Knops, H.(eds.), IOS Press, pp. 302-307, 1999.

[8] T. Horvath, T. Abdelzaher, K. Skadron, and L. Xue, "Dynamic

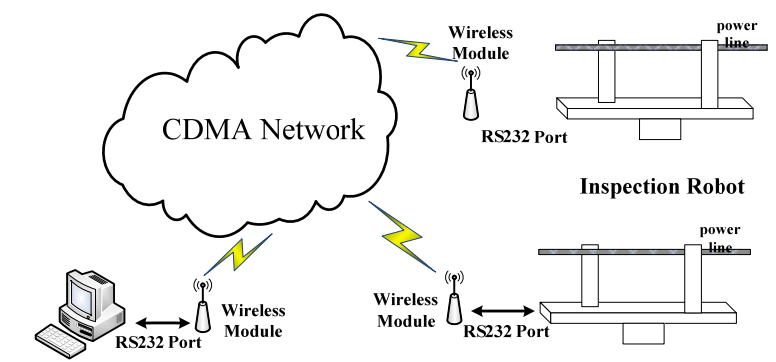

Maintenance Center

Server

Figure 1. Remote maintenance system architecture

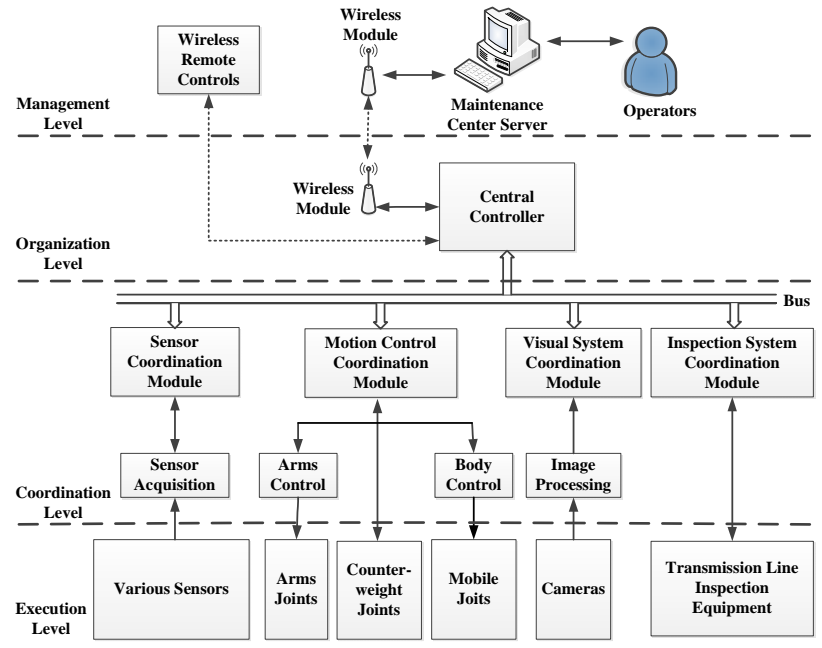

Figure 2. Inspection robot control block diagram

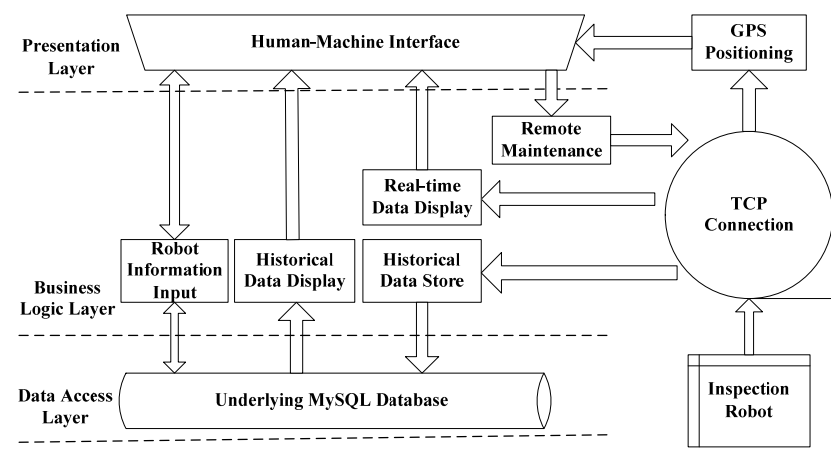

Figure 3. Remote maintenance software block diagram
Voltage Scaling in Multitier Web Servers with End-to-End Delay Control," Computers, IEEE Transactions on, vol. 56, pp. 444-458, 2007.

[9] Wikipedia. Network address translation. Available: http://en.wikipedia.org/wiki/Network_address_translation

[10] L. Bo, "Analysis of New Rebound Port Trojan Horse, Gwboy," Journal of Changsha University of Electric Power (Natural Science), vol. 1, 2005.

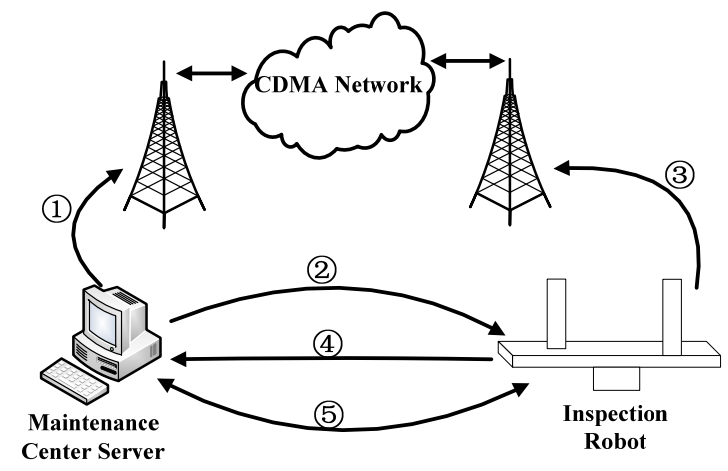

Figure 4. Networking method

\begin{tabular}{|c|c|c|}
\hline Header & Frame Data & End \\
\hline
\end{tabular}

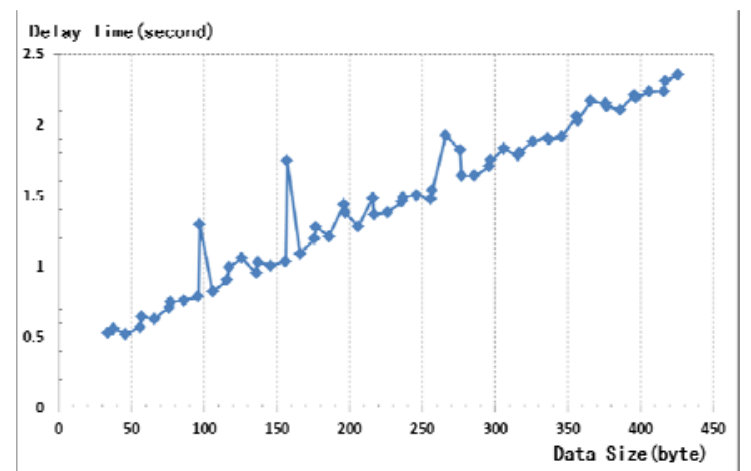

Figure 6. Communication delay statistics

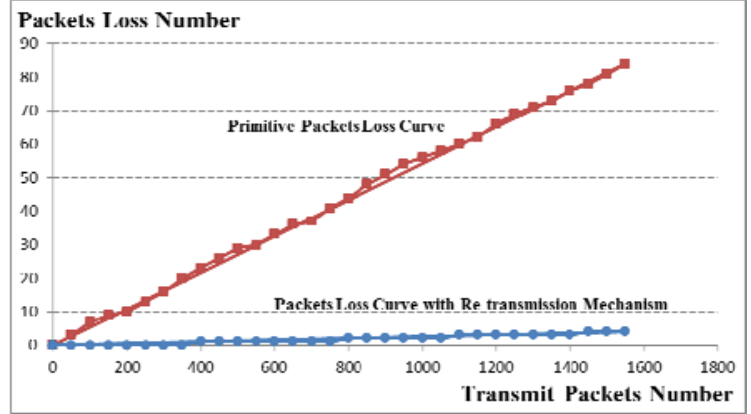

Figure 7. Data communication packet loss statistics 